\title{
Influence Research of Thermal Power Unit's Extraction Steam for Power Grid Frequency Modulation
}

\author{
Jun $\mathrm{Li}^{1, \text { a }}$, Hui Zhang ${ }^{2, \mathrm{~b}}$ and Meng Wang ${ }^{1, \mathrm{c}}$ \\ ${ }^{1}$ Shandong Electric Power Research Institute, Jinan 250002, China \\ ${ }^{2}$ Dispatching \& Control Center, Shandong Electric Power Corporation, Jinan 250001, China \\ alijun_sdu@hotmail.com, bzhanghui84@163.com, cwangmeng339@gmail.com
}

Keywords: frequency modulation, thermal power unit, extraction steam, thermodynamic model Abstract. Wind power and other new energy connected to power grid bring new challenges to optimal operation of power systems. The stability of power grid mainly depends on primary frequency compensation and secondary frequency compensation of thermal power units. But thermal power unit common have extraction conditions. In the analysis of thermodynamic system of thermal power unit, the relationship between thermal power extraction steam and unit's load has analyzed and calculated. The practical application results show that extraction affecting power capability of the unit, is not conducive to adjust the grid frequency.

\section{Introduction}

In recent years, the proportion of a heating unit in China is increasing. The thermoelectric tends to cause large-scale development. To make full use of the advantages of cogeneration, large capacity over $300 \mathrm{mw}$ condensing steam turbine as examples of a heating unit increased year by year, but because of its heat extraction for the adjustment of extraction, mostly for heating medium quality requirements higher heating transformation, generally uses the reheater hot period of extraction steam heating or reheater cold period of extraction steam heating, after desuperheating decompression device supply heat users. Extraction steam can cause total steam flow is insufficient, finally affects the frequency modulation of the unit load capacity.

As is well known, power grid frequency is one of the three indicators of power quality, it reflects the balance between the generation of active power and load, and it is an important control parameter of the power system operation. The safety and efficiency of the majority of users of electrical equipment and power equipment have a close relationship with power grid frequency. Equipment of users is generally driven by the motor, the same as equipment of power plant. The frequency fluctuation has a critical influence over them. With the development of science and technology, some new electronic equipment and precision processing equipment put forward higher request to power grid frequency, the frequency fluctuations will lead to lower product quality or equipment damage. According to the IEEE $446-1995$ standard, $\pm 0.5 \mathrm{~Hz}$ is the maximum tolerance of the frequency fluctuations of many devices. The occurrence of unexpected failures in the power grid will break the balance between the generation of active power and load. With the development of the power system, single device failures bring a growing loss of generating power grid. Only relying on manually adjust the power generation output will require a longer time to achieve a new balance. To solve these problems, the way is only one that is the techniques of primary frequency compensation (PFC) and automatic generation control (AGC, the equivalent of secondary FC). It's a good idea to use different logical controls according to its own regulation performance, to maintain the balance of power system supply and demand automatically, thus ensuring the quality of the power system frequency.

\section{Thermodynamic Model}

Coefficient calculation method. The heating unit is designed according to the first design principles, assumptions: new steam pressure, each extraction pressure, back pressure are constant. Each cylinder back pressure change affects the quantity of admission is neglected, so that each cylinder inlet steam flow completely controlled by the cylinder inlet steam valves. Assumes that flow along with the 
change of the opening of the each regulator is linear, the corresponding power of each cylinder also along with the change of flow rate is linear. High, medium and low oil motivations have the same time constant.

The basic calculation method as follows,

According to each flow valve lift curve, we can obtain various valve opening and the flow rate, the relationship between and maximum of the opening of the tone of the steam flow is obtained $\mathrm{D}_{10}, \mathrm{D}_{20}$ and D30. According to thermodynamic calculations control or operating mode chart, get the corresponding valve flow of each cylinder power, is obtained $\mathrm{N}_{10}, \mathrm{~N}_{20}$ and $\mathrm{N}_{30}$. According to the above calculated the amount of each cylinder of steam and the corresponding power, coupling coefficient obtained directly. According to the working condition of typical thermal equilibrium diagram, select two typical working conditions, and under the conditions of tone than lift or flow, plug in the power and the calculation formula of the amount of extraction set up simultaneous equations, and the coupling coefficient. After the coupling coefficient obtained, by solving the determinant, decoupling coefficient can be calculated.

Fundamental relations. According to assumptions, single pumping unit is equivalent to a back-pressure machine and a pure condensate coaxial machine. The schematic diagram of single exhaust steam turbine is show in Fig.1.

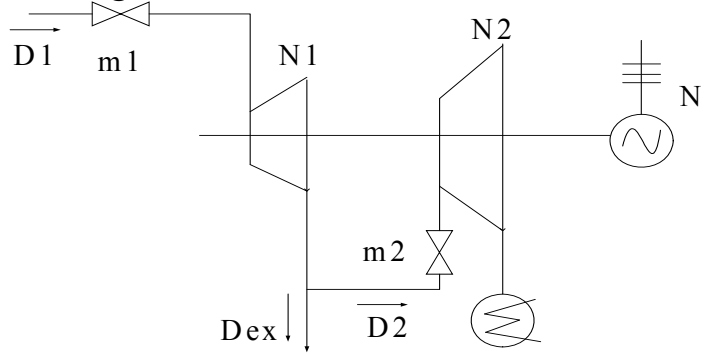

Fig.1 Single exhaust steam turbine schematic diagram

Accompanying single exhaust steam turbine regulating system diagram is shown in Fig.2.

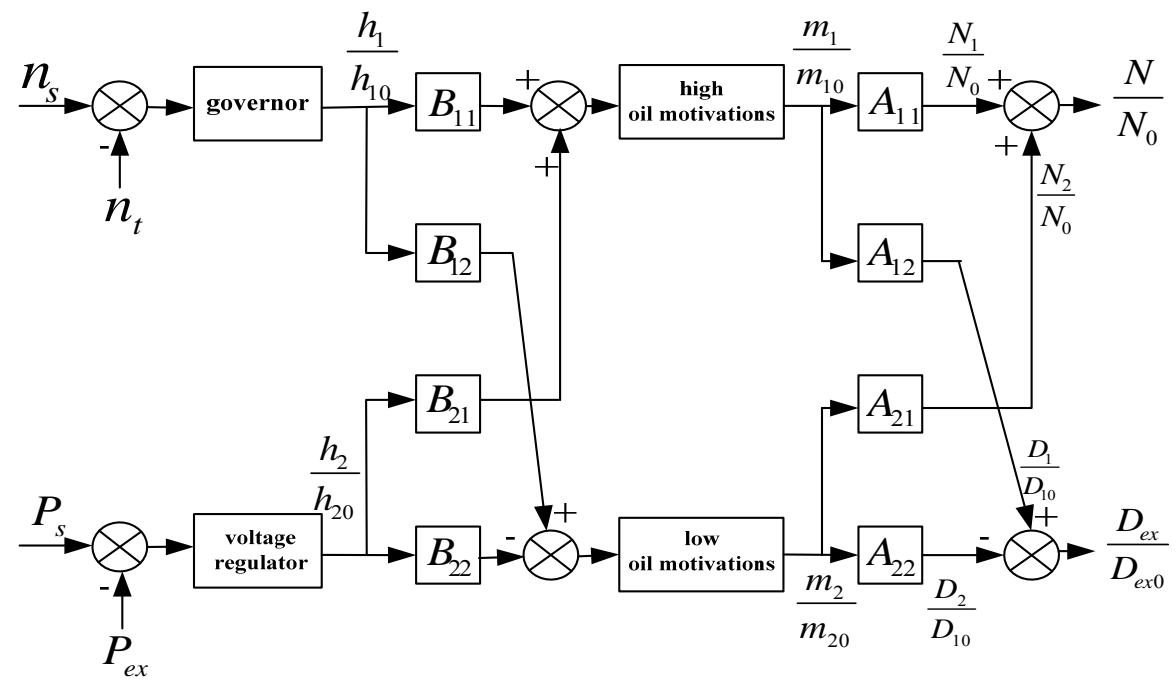

Fig.2 Single exhaust steam turbine regulating system diagram

Power and extraction quantity relation

$$
\left\{\begin{array}{l}
N=N_{1}+N_{2} \\
D_{e x}=\alpha\left(D_{1}-D_{2}\right)
\end{array}\right.
$$

$\alpha$ is the coefficient is less than 1 , to consider the influence of the amount of heat extraction on extraction

$$
\alpha=\frac{D_{e x 0}}{\left(D_{1}-D_{2}\right)_{0}}
$$

Linear power, flow regulating valve opening characteristic 


$$
\left\{\begin{array}{l}
N_{1} \propto D_{1} \propto m_{1} \\
N_{2} \propto D_{2} \propto m_{2}
\end{array}\right.
$$

By the high pressure adjusting value control unit's acceleration, interconnection, load, until with full load. The host extraction point should have been higher than the rated pressure extraction steam pressure, meet with heat load conditions.

\section{Frequency Regulation}

Frequency regulation of power system is inherent in the use of system load frequency characteristics, as well as the role of the generator's governor to prevent the system frequency deviation from the standard adjustment method. The grid frequency is determined by the value of generated energy and electricity consumption. If the value of generated energy is equal to the electricity consumption, the grid frequency is stably. If the value of generated energy is greater than the electricity consumption, the grid frequency will increase, otherwise, the grid frequency will reduce.

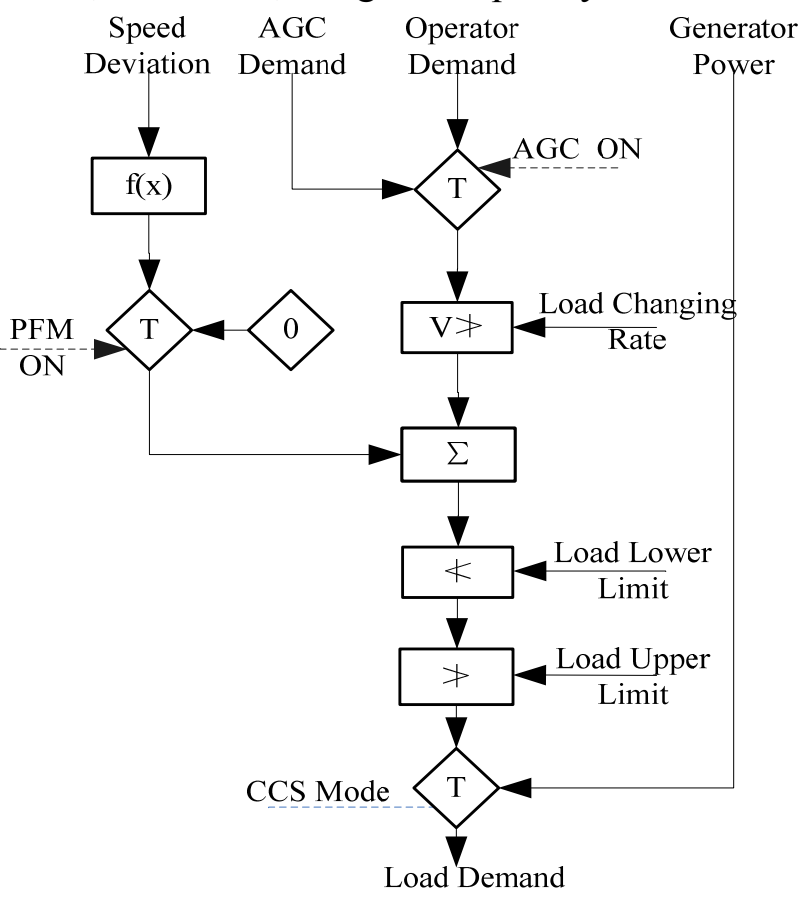

Fig.3 Logic of PFC and AGC in CCS side

Generally speaking, the PFC in domestic most power plants adopts coordinated control system (CCS) and DEH control strategy. DEH is implementation stage, difference, open loop control, ensure frequency compensation's rapidity, CCS is correction stage, no difference, closed loop control, ensure the accuracy and persistent of frequency compensation. In order to guarantee the quality of regulation, speed and power signal of PFC have been done by selecting mid-valve of three same signals, to be shared with DEH and CCS system, to ensure the consistency of judgment on both sides of the signal. As shown in Fig.3, speed deviation signal is converted to the corresponding frequency modulation power through the function generator $\mathrm{f}(\mathrm{x})$, and added to the unit load after rate limiting, and after load high and low limit, forms the final actual load demand, ensure frequency compensation demand quickly and correctly and accurately.

\section{Actual Operation}

For example, in one $350 \mathrm{MW}$ subcritical coal-fired power plant, its total steam flow is $1058 \mathrm{t} / \mathrm{h}$. The design max extraction steam is $238 \mathrm{t} / \mathrm{h}$. Because the extraction steam in winter, unit load cannot reach the max value $350 \mathrm{MW}$ max value. Some comparison data of extraction unit is shown in Table 1. 
Table 1 Extraction steam and unit load comparison data

\begin{tabular}{ccccccc}
\multirow{2}{*}{$\begin{array}{c}\text { Working } \\
\text { condition }\end{array}$} & \multicolumn{3}{c}{ No Extraction steam } & \multicolumn{3}{c}{ Extraction steam } \\
\cline { 2 - 6 } & $\begin{array}{c}\text { Unit } \\
\text { load }\end{array}$ & $\begin{array}{c}\text { Total steam } \\
\text { flow }\end{array}$ & $\begin{array}{c}\text { Extraction } \\
\text { steam }\end{array}$ & $\begin{array}{c}\text { Unit } \\
\text { load }\end{array}$ & $\begin{array}{c}\text { Total steam } \\
\text { flow }\end{array}$ & $\begin{array}{c}\text { Extraction } \\
\text { steam }\end{array}$ \\
\hline 1 & 200.07 & 596.07 & 0.00 & 200.08 & 686.76 & 184.01 \\
2 & 250.02 & 737.58 & 0.00 & 250.08 & 800.44 & 163.56 \\
3 & 280.08 & 837.83 & 0.00 & 280.05 & 1005.62 & 287.54 \\
4 & 300.05 & 914.29 & 0.00 & 300.02 & 1023.33 & 221.77 \\
\hline
\end{tabular}

In actual operation, when the extraction steam of unit is $200 \mathrm{t} / \mathrm{h}$, the max unit load can only reach $300 \mathrm{MW}$. When the unit load is more than $300 \mathrm{MW}$, the frequency modulation is ability cannot meet the requirement of the power grid frequency modulation.

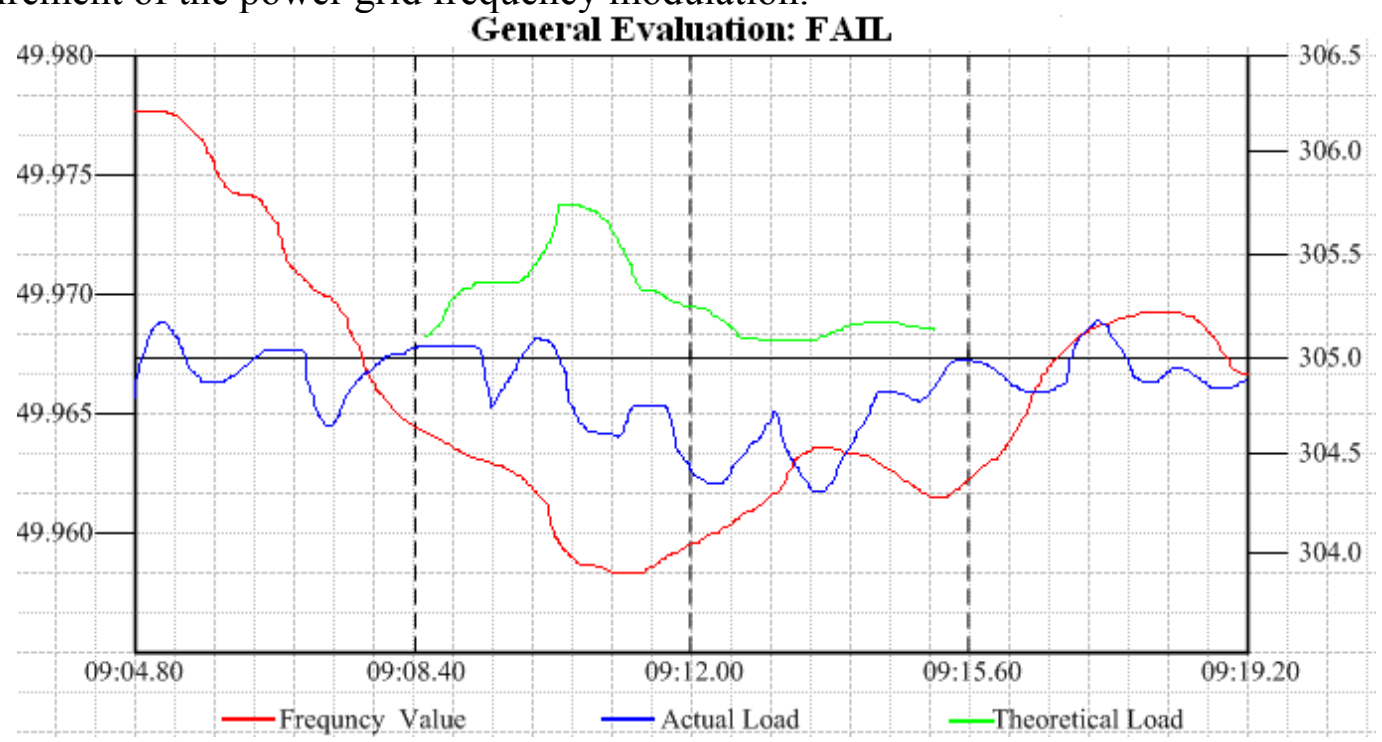

Fig.4 Unqualified Frequency modulation curve

\section{Conclusions}

By analyzing thermodynamic system of thermal power unit, primary frequency compensation control strategy response to frequency of accidents and the relationship between thermal power extraction steam and unit's load, points out that the extraction affects the actual work ability of the unit. It is not conducive to adjust the grid frequency. The practical operation results that in order to the stability of power grid frequency, the corresponding control measures must be taken.

\section{References}

[1] Karl J.Astrom, and Tore Hagglund, Advanced PID Control, ISA, (2006)70-73.

[2] D.R.Coyghanowr, Process Systems Analysis and Control, McGraw-Hill, New York, 1991.

[3] Yang Wei-guang, LU Ai, LI Xue-gang, "Study on peak regulation calculation scheme for on-line monitoring system of heat supplying units", Jilin Electric Power, 2010, 38(6), pp.5-7.

[4] M. Zhuang, D.P. Atherton, Automatic tuning of optimum PID controllers, IEE ProcD, 140, 1993, pp. 216-224.

[4] Ma Jian-wei, Ge Ting, Jing Bai-lin, et al, "Research and practice for energy-saving power generation and dispatching of cogeneration power unit", Electric Power, 2009, 42(4), pp.14-17.

[5] Xie Chun-lin, XueYong-feng, WANG Song-ling, "Research on the model of ordering power by heat in on-line monitoring system of heat-supply turbine units", Northeast Electric Power Technology, 2012(7), pp.9-11. 